\title{
Prophylactic Statin Administration May Prevent Shortening of the Fibrillation Cycle Length in Patients With New-Onset Atrial Fibrillation
}

\author{
Jun Oikawa, ${ }^{1}$ MD, Shinichi Niwano, ${ }^{1} \mathrm{MD}$, Hiroe Niwano, ${ }^{2} \mathrm{MD}$, Naruya Ishizue, ${ }^{1} \mathrm{MD}$, \\ Tomoharu Yoshizawa, ${ }^{1} \mathrm{MD}$, Akira Satoh,,${ }^{1} \mathrm{MD}$, Sayaka Kurokawa, ${ }^{1} \mathrm{MD}$, \\ Yuko Hatakeyama, ${ }^{1} \mathrm{MD}$, and Hidehira Fukaya, ${ }^{1} \mathrm{MD}$
}

\begin{abstract}
SUMMARY
Patients with recently diagnosed atrial fibrillation (AF) tend to exhibit a longer fibrillation cycle length (FCL) than those having a longer clinical history. However, the electrophysiological properties of new-onset AF may vary because of the clinical background of patients. In this study, we evaluated clinical factors to identify the determinants of FCL in new-onset AF. Electrocardiograms (ECGs) recorded from 2008 through 2011 were analyzed using our digital ECG-profiling system. In the 1,578 AF episodes recorded, 466 new-onset AF episodes were identified using clinical referral history and previous ECGs. After evaluating FCL in these new-onset AF episodes, using a customized fibrillation wave analyzer with fast Fourier transform analysis, we divided the patients into a longer-FCL group and a shorter-FCL group using the median FCL (158 ms). Propensity score matching yielded 135 matched pairs of patients with comparable mean ages between the two groups. Four factors (brain natriuretic peptide levels, and use of angiotensin receptor blockers, calcium channel blockers or statins) exhibited a significant difference between the two groups. Multivariate analysis revealed that statin use was the only significant independent predictor of longer FCL (Odds ratio, 3.86; 95\% CI, 1.659.63; $P=0.003)$. Among various clinical parameters, statin use was related to longer FCL at the time of new-onset AF in patients with AF. (Int Heart J 2013; 54: 371-376)
\end{abstract}

Key words: Upstream therapy, Fast fourier transform

A trial fibrillation (AF) is one of the most common arrhythmias in clinical practice, ${ }^{1)}$ and it is a major cause of morbidity and mortality in the general population. ${ }^{2)}$ The prevalence of AF is related to aging, and there are approximately 716,000 people with AF in Japan, an overall prevalence of $0.56 \%$ in the Japanese population. ${ }^{3)}$ The arrhythmogenic substrate of AF is known to be constructed progressively under various pathological conditions, including AF itself. This process is understood as atrial remodeling. Therapies designed to suppress this remodeling are termed "upstream therapy." Although the suppressive effects of some medicines, such as angiotensin receptor blockers (ARBs) ${ }^{4,5)}$ or statins, ${ }^{6,7)}$ as upstream therapy have been experimentally documented in animal models of AF, the clinical effect of this therapy is still controversial. ${ }^{8-10)}$ The reason for this discrepancy between experimental and clinical results remains unclear, but the atrial structural changes may have already progressed further in clinical cases than in experimental models, even in cases with paroxysmal AF, because of the influence of various clinical fac- tors. We evaluated the atrial electrophysiological properties through analysis of the fibrillation cycle length (FCL). ${ }^{11)}$ This method enables us to evaluate the degree of refractory shortening or the complexity of the AF substrate noninvasively and repeatedly, and then gives us indirect but important information about the degree of atrial remodeling. ${ }^{12)}$ In the present study, we hypothesized that various clinical factors including those related to the disease underlying $\mathrm{AF}$ and/or the use of various prescribed medicines, may influence atrial electrophysiological properties even before the clinical appearance of AF. These clinical factors were compared between patients with longer and shorter FCL at the time of new-onset AF to identify the factors that may influence FCL.

\section{MeTHODS}

Study population: During 2008-2011, a total of 83,847 12lead electrocardiogram (ECG) recordings were stored in Kita-

From the ${ }^{1}$ Department of Cardio-Angiology, Kitasato University School of Medicine, Sagamihara, and ${ }^{2}$ Department of Education, Tamagawa University, College of Education, Machida, Japan.

This study was supported by a grant for scientific research from the Ministry of Education Science and Culture of Japan (No.11838015), a grant to the Research Committee for Epidemiology and Etiology of Idiopathic Cardiomyopathy from the Ministry of Health and Welfare of Japan, and a Kitasato University Research Grant for Young Researchers.

Address for correspondence: Jun Oikawa, MD, Department of Cardio-Angiology, Kitasato University School of Medicine, 1-15-1 Kitasato, Minami-ku, Sagamihara 252-0374, Japan.

Received for publication May 31, 2013.

Revised and accepted June 17, 2013. 
sato University Hospital's digital ECG profiling system. From these recordings, we identified 1,578 patients from 8,543 AF episodes. Patients with an available ECG recording within the 3 months preceding their AF episode were selected for the study. By referring to their clinical history of suspicious symptoms, such as palpitations, and preceding ECG recordings, 1,578 AF episodes of 466 patients were identified as new-onset AF (Figure 1). Patients taking class I or class III antiarrhythmic agents were excluded because a previous study reported that these agents prolonged FCL. ${ }^{13,14)}$ The mean age of these 466 patients was $69 \pm 12$ years, and 152 were female. There was no apparent underlying disease in 111 of the AF patients, however, patients with underlying diseases, such as congestive heart failure, valvular heart disease, diabetes mellitus, and hypertension were included. All studies were conducted with the approval of the Ethics Committee of Kitasato University Hospital and written informed consent was obtained before analyzing the data.

Analysis of the fibrillation wave: Spectral analysis was performed using data from the surface ECG recording in lead V1, which was extracted from digitally stored data in the ECG profiling server. The QRS-T complex was subtracted, using a template-matching algorithm, to obtain the pure fibrillation wave for analysis. ${ }^{15)}$ The frequency analysis of the subtracted ECG involved 3 steps: (1) band-pass filtering, (2) application of a Hamming window, and (3) 1024-point fast Fourier transformation. The $50 \%$ overlap of adjacent spectral analyses allowed the use of an average of 8 epochs of analysis within a single 8 s data set. After spectral analysis, the power spectra were quantified by measuring the peak frequency signal with the maximum magnitude derived from the epoch. The peak frequency of the spectrum in the $3-12 \mathrm{~Hz}$ range was converted to a cycle length (CL in $\mathrm{ms}=1000 /$ frequency in $\mathrm{Hz}$ ), defined as the fibrillation cycle length (FCL), and was calculated as an average of 8 epochs.

Grouping and comparison of data: The 466 patients were divided into two groups, a longer-FCL group and shorter-FCL group, using the median FCL as the boundary. We then compared FCL and clinical data between the two groups. The presence or absence of cardiovascular disease was evaluated by echocardiogram and cardiac catheterization in selected cases.

Our primary analysis was a comparison of the patient's clinical factors between the longer- and shorter-FCL groups. To further control potential confounding and selection bias, we created a propensity-matched model using the propensity score matching technique. ${ }^{16)}$ For creating this model, a propensity score is calculated for each individual on the bias of background covariates. A propensity score for each longer-FCL patient was then calculated from their logistic equation. Specifically, we sought to match each longer-FCL patient to a shorterFCL patient who had a propensity score that was identical to 4 digits. Once this threshold was exceeded, those longer- and shorter-FCL patients were excluded. Finally, we could match 135 longer-FCL patients to 135 shorter-FCL patients (Figure $1)$.

Statistical analysis: Statistical analysis was performed using the JMP 9 (SAS Japan Inc, Tokyo) statistical software package. Continuous variables are presented as the mean \pm standard deviation and were compared using the Student $t$ test or MannWhitney $U$ test. Dichotomous variables are presented as percentages and were compared using the $\chi^{2}$ test. A $P$ value of $<$

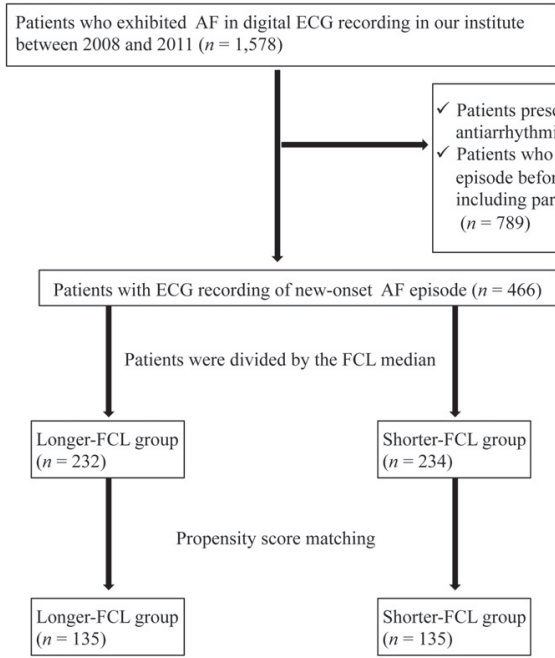

Figure 1. Derivation of the study population. We selected atrial fibrillation (AF) patients whose ECG had been recorded between 2008 and 2011. There were two exclusion criteria. (1) patients prescribed class I or III antiarrhythmic agents, and (2) patients with evidence of AF before 2008, including paroxysmal or persistent AF. The 466 patients selected were divided into two groups by the median FCL. Finally, we used propensity score matching to create two age-adjusted patient groups.

0.05 was considered to be statistically significant.

\section{Results}

Comparison of patients with longer and shorter FCL: From a pool of 466 patients, 232 patients were assigned to the longerFCL group and 234 patients were assigned to the shorter-FCL groups. The mean FCL was $165 \pm 34 \mathrm{~ms}$ (range, 111-379 ms) and the median was $158 \mathrm{~ms}$. The mean age was $71 \pm 12$ years for the longer-FCL group and $66 \pm 12$ years for the shorterFCL group $(P<0.0001)$. Comparison of the longer- and shorter-FCL groups revealed several clinical factors that exhibited significant differences between them, including age, serum levels of brain natriuretic peptide (BNP) and low-density lipoprotein (LDL) cholesterol, prevalence of hypertension and smoking, and use of angiotensin receptor blockers (ARBs)/angiotensin converting enzyme inhibitors (ACEIs), calcium channel blockers, and statins (Tables I and II).

Comparison in propensity-matched patients: After propensity score matching, there were 135 matched pairs of patients with longer and shorter FCL. The mean age was comparable between the two groups (Tables III and IV). The mean age of the study population was $68 \pm 11$ years, and $31.9 \%$ of the patients were female. Oral anticoagulation therapy using vitamin $\mathrm{K}$ antagonists was administered in $24 \%$ of the patients, and the mean $\mathrm{CHADS}_{2}$ score was 1.7 points. The mean FCL was 186 $\pm 34 \mathrm{~ms}$ in the longer-FCL group and $143 \pm 11 \mathrm{~ms}$ in the shorter-FCL group. The clinical characteristics of the longerand shorter-FCL patients are summarized in Tables III and IV. When comparing the two groups, no clinical background parameter, including gender, heart rate, blood pressure, echocardiographic parameters or prevalence of underlying diseases exhibited a significant difference. In contrast, the incidence of 
Table I. Clinical Characteristics of Patients With Longer- and Shorter-FCL

\begin{tabular}{|c|c|c|c|c|}
\hline & $\begin{array}{c}\text { Total } \\
(n=466)\end{array}$ & $\begin{array}{l}\text { Longer-FCL group } \\
\qquad(n=232)\end{array}$ & $\begin{array}{l}\text { Shorter-FCL group } \\
\quad(n=234)\end{array}$ & $P$ \\
\hline Mean FCL (ms) & $165 \pm 34$ & $188 \pm 34$ & $143 \pm 11$ & \\
\hline Age & $69 \pm 12$ & $71 \pm 12$ & $66 \pm 12$ & $<0.0001^{*}$ \\
\hline Gender (M : F) & $314: 152$ & $147: 85$ & $167: 67$ & 0.07 \\
\hline Heart rate (bpm) & $90 \pm 25$ & $92 \pm 28$ & $88 \pm 23$ & 0.18 \\
\hline $\mathrm{sBP}(\mathrm{mmHg})$ & $129 \pm 22$ & $128 \pm 22$ & $131 \pm 22$ & 0.17 \\
\hline $\mathrm{dBP}(\mathrm{mmHg})$ & $77 \pm 15$ & $76 \pm 17$ & $79 \pm 14$ & 0.05 \\
\hline \multicolumn{5}{|l|}{ Echocardiographic data } \\
\hline $\mathrm{LAD}(\mathrm{mm})$ & $47 \pm 9$ & $47 \pm 10$ & $47 \pm 7$ & 0.97 \\
\hline $\operatorname{LVEF}(\%)$ & $60 \pm 12$ & $61 \pm 11$ & $60 \pm 13$ & 0.55 \\
\hline \multicolumn{5}{|l|}{ Laboratory data } \\
\hline $\mathrm{BNP}(\mathrm{pg} / \mathrm{mL})$ & $306 \pm 426$ & $355 \pm 492$ & $257 \pm 340$ & $0.03 *$ \\
\hline eGFR (mL/minute) & $65 \pm 26$ & $63 \pm 26$ & $67 \pm 26$ & 0.14 \\
\hline $\mathrm{UA}(\mathrm{mg} / \mathrm{dL})$ & $6.3 \pm 2.3$ & $6.4 \pm 2.8$ & $6.2 \pm 1.6$ & 0.37 \\
\hline LDL cholesterol (mg/dL) & $109 \pm 37$ & $105 \pm 35$ & $113 \pm 38$ & $0.03 *$ \\
\hline $\operatorname{HbAlc}(\%)$ & $6.1 \pm 1.2$ & $6.1 \pm 1.2$ & $6.2 \pm 1.3$ & 0.84 \\
\hline $\mathrm{TSH}(\mu \mathrm{IU} / \mathrm{mL})$ & $2.45 \pm 3.0$ & $2.30 \pm 2.8$ & $2.27 \pm 2.2$ & 0.94 \\
\hline FT3 (pg/mL) & $3.09 \pm 2.1$ & $3.69 \pm 4.9$ & $4.53 \pm 6.0$ & 0.31 \\
\hline FT4 (ng/dL) & $1.47 \pm 0.9$ & $1.69 \pm 1.4$ & $1.81 \pm 1.6$ & 0.56 \\
\hline
\end{tabular}

sBP indicates systolic blood pressure; dBP, diastolic blood pressure; LAD, left atrial dimension; LVEF, left ventricular ejection fraction; BNP, brain natriuretic peptide, UA, urea acid; LDL cholesterol, low-density lipoprotein cholesterol; and TSH, thyroid-stimulating hormone.

Table II. Underlying Disease and Incidence of Prescription in Patients With Longer- and Shorter-FCL

\begin{tabular}{lcccc}
\hline & $\begin{array}{c}\text { Total } \\
(n=466)\end{array}$ & $\begin{array}{c}\text { Longer-FCL group } \\
(n=232)\end{array}$ & $\begin{array}{c}\text { Shorter-FCL group } \\
(n=234)\end{array}$ & $P$ \\
\hline Underlying disease & & & & \\
$\quad$ Congestive heart failure & $84(18 \%)$ & $47(20 \%)$ & $37(19 \%)$ & 0.22 \\
$\quad$ Valvular heart disease & $118(25 \%)$ & $62(27 \%)$ & $56(24 \%)$ & 0.18 \\
$\quad$ Hypertension & $293(62 \%)$ & $155(67 \%)$ & $138(59 \%)$ & $0.04 *$ \\
$\quad$ Diabetes mellitus & $129(28 \%)$ & $68(29 \%)$ & $61(26 \%)$ & 0.47 \\
Incidence of prescription & $180(39 \%)$ & $101(44 \%)$ & $79(34 \%)$ & $0.02 *$ \\
ARBs/ACEIs & $112(24 \%)$ & $62(27 \%)$ & $50(21 \%)$ & 0.13 \\
$\quad \beta$-Blockers & $129(28 \%)$ & $80(34 \%)$ & $49(21 \%)$ & $0.0006 *$ \\
Calcium-channel blockers & $80(17 \%)$ & $48(21 \%)$ & $32(14 \%)$ & $0.03 *$ \\
Statins & $76(16 \%)$ & $29(13 \%)$ & $47(20 \%)$ & $0.01 *$ \\
\hline Smoking & & & \\
\hline
\end{tabular}

$\mathrm{ARB}$ indicates angiotensin receptor blocker and ACEI, angiotensin converting enzyme inhibitor.

prescription of ARBs/ACEIs ( $50 \%$ versus $35 \%, P=0.02$ ), calcium channel blockers ( $40 \%$ versus $23 \%, P=0.005$ ), and statins $(29 \%$ versus $14 \%, P=0.002)$ was higher in the longerFCL group than in the shorter-FCL group. Serum BNP level was also higher in the longer-FCL $(P=0.01)$.

Multivariate analysis: Table V summarizes the results of a multivariate analysis using the prevalence of underlying diseases and parameters that exhibited a significant difference between the two groups in the univariate analysis (ie, BNP level and use of ARBs/ACEIs, calcium channel blockers, and statins). The multivariate analysis revealed that the use of statins was the only significant independent parameter for predicting longer and shorter FCL (Odds ratio, 3.87; 95\%CI, 1.65-9.65; $P$ $=0.002$ ).

Comparison of patients with and without statins: The patients in this study used several different statins, including pravastatin $(31 \%)$, atorvastatin $(24 \%)$, pitavastatin $(18 \%)$, rosuvastatin $(15 \%)$, simvastatin $(11 \%)$, and fluvastatin $(1 \%)$. When comparing the longer- and shorter- FCL groups, there was no difference in the distribution of statin use. Because the multivariate analysis revealed that the use of statins was an independent determinant for longer FCL in new-onset AF, we compared patients with and without statin prescription, after age adjustment, using propensity score matching. We found that patients with statin prescriptions exhibited significantly longer FCL than patients without statin prescriptions $(P<0.0001$, Figure 2).

\section{Discussion}

The present study, which detected patients with new-onset AF ECG recording and analyzed various clinical parameters including FCL in the surface ECG, has several interesting findings. First, this was the first systematic trial of detection of new-onset AF, and 466 patients with new-onset AF were identified from the 4-year database comprising 83,847 digital ECG recordings. Second, our customized fibrillation wave analyzer could demonstrate mean and median FCL of $165 \mathrm{~ms}$ and 158 $\mathrm{ms}$ in the population of new-onset AF. Third, the incidence of prescription of calcium channel blockers, ARBs/ACEIs, or statins, as well as serum BNP level exhibited significant differ- 
Table III. Clinical Characteristics of the Patients With Longer- and Shorter-FCL (Propensity Score Matching)

\begin{tabular}{|c|c|c|c|c|}
\hline & $\begin{array}{c}\text { Total } \\
(n=270)\end{array}$ & $\begin{array}{l}\text { Longer-FCL group } \\
\qquad(n=135)\end{array}$ & $\begin{array}{l}\text { Shorter-FCL group } \\
\quad(n=135)\end{array}$ & $P$ \\
\hline Mean FCL (ms) & $164 \pm 33$ & $186 \pm 34$ & $143 \pm 11$ & \\
\hline Age & $68 \pm 11$ & $68 \pm 11$ & $68 \pm 11$ & 1.0 \\
\hline Gender (M : F) & $184: 86$ & $91: 44$ & $93: 42$ & 0.79 \\
\hline Heart rate (bpm) & $92 \pm 25$ & $93 \pm 28$ & $90 \pm 22$ & 0.33 \\
\hline $\mathrm{sBP}(\mathrm{mmHg})$ & $131 \pm 23$ & $131 \pm 22$ & $132 \pm 23$ & 0.66 \\
\hline $\mathrm{dBP}(\mathrm{mmHg})$ & $77 \pm 16$ & $78 \pm 14$ & $77 \pm 17$ & 0.41 \\
\hline \multicolumn{5}{|l|}{ Echocardiographic data } \\
\hline $\operatorname{LAD}(\mathrm{mm})$ & $47 \pm 8$ & $47 \pm 8$ & $47 \pm 7$ & 0.61 \\
\hline $\operatorname{LVEF}(\%)$ & $60 \pm 12$ & $60 \pm 11$ & $60 \pm 13$ & 0.44 \\
\hline \multicolumn{5}{|l|}{ Laboratory data } \\
\hline $\mathrm{BNP}(\mathrm{pg} / \mathrm{mL})$ & $300 \pm 417$ & $350 \pm 511$ & $251 \pm 295$ & $0.01 *$ \\
\hline eGFR (mL/minute) & $65 \pm 24$ & $63 \pm 28$ & $64 \pm 21$ & 0.94 \\
\hline $\mathrm{UA}(\mathrm{mg} / \mathrm{dL})$ & $6.2 \pm 2.6$ & $6.4 \pm 3.3$ & $6.1 \pm 1.7$ & 0.95 \\
\hline LDL cholesterol (mg/dL) & $110 \pm 38$ & $105 \pm 36$ & $115 \pm 40$ & 0.06 \\
\hline $\operatorname{HbAlc}(\%)$ & $6.3 \pm 1.3$ & $6.2 \pm 1.1$ & $6.3 \pm 1.4$ & 0.59 \\
\hline TSH ( $\mu \mathrm{IU} / \mathrm{mL})$ & $2.27 \pm 2.4$ & $2.02 \pm 2.1$ & $2.48 \pm 2.7$ & 0.43 \\
\hline FT3 $(\mathrm{pg} / \mathrm{mL})$ & $4.23 \pm 5.8$ & $4.24 \pm 6.2$ & $4.21 \pm 5.4$ & 0.06 \\
\hline FT4 (ng/dL) & $1.81 \pm 1.6$ & $1.89 \pm 1.8$ & $1.74 \pm 1.4$ & 0.89 \\
\hline
\end{tabular}

sBP indicates systolic blood pressure; $\mathrm{dBP}$, diastolic blood pressure; LAD, left atrial dimension; LVEF, left ventricular ejection fraction; BNP, brain natriuretic peptide, UA, urea acid; LDL cholesterol, low-density lipoprotein cholesterol; and TSH, thyroid-stimulating hormone.

Table IV. Underlying Disease and Incidence of Prescription in Longer- and Shorter-FCL Patients (Propensity Score Matching)

\begin{tabular}{lcccc}
\hline & $\begin{array}{c}\text { Total } \\
(n=270)\end{array}$ & $\begin{array}{c}\text { Longer-FCL group } \\
(n=135)\end{array}$ & $\begin{array}{c}\text { Shorter-FCL group } \\
(n=135)\end{array}$ & $P$ \\
\hline Under lying disease & & & & \\
$\quad$ Congestive heart failure & $55(21 \%)$ & $31(30 \%)$ & $24(18 \%)$ & 0.32 \\
$\quad$ Valvular heart disease & $74(32 \%)$ & $38(35 \%)$ & $36(29 \%)$ & 0.39 \\
Hypertension & $171(64 \%)$ & $91(68 \%)$ & $80(60 \%)$ & 0.16 \\
Diabetes mellitus & $75(30 \%)$ & $41(32 \%)$ & $34(27 \%)$ & 0.38 \\
Incidence of prescription & $110(42 \%)$ & $64(50 \%)$ & $46(35 \%)$ & $0.02 *$ \\
ARBs/ACEIs & $70(27 \%)$ & $40(31 \%)$ & $30(23 \%)$ & 0.13 \\
$\beta$-Blockers & $82(31 \%)$ & $51(40 \%)$ & $31(23 \%)$ & $0.005 *$ \\
Calcium-channel blockers & $55(21 \%)$ & $37(29 \%)$ & $18(14 \%)$ & $0.002 *$ \\
Statins & $42(33 \%)$ & $21(31 \%)$ & $21(36 \%)$ & 0.53 \\
Smoking & . & & & \\
\hline
\end{tabular}

$\mathrm{ARB}$ indicates angiotensin receptor blocker and ACEI, angiotensin converting enzyme inhibitor.

Table V. Multivariate Analysis of the Clinical Parameters (Laboratory Data, Underlying Disease, Incidence of Prescription)

\begin{tabular}{lccc}
\hline & Odds ratio & $95 \%$ CI \\
\hline Underlying disease & & & $P$ \\
$\quad$ Congestive heart failure & 1.2561 & $0.533-2.935$ & 0.60 \\
$\quad$ Valvular heart disease & 1.1971 & $0.571-2.499$ & 0.63 \\
$\quad$ Hypertension & 0.9311 & $0.356-2.389$ & 0.88 \\
$\quad$ Diabetes mellitus & 0.9301 & $0.449-1.903$ & 0.84 \\
Incidence of prescription & & & 0.54 \\
$\quad$ ARBs/ACEIs & 0.7544 & $0.305-1.835$ & 0.65 \\
$\quad \beta$-Blockers & 0.8228 & $0.346-1.923$ & 0.06 \\
$\quad$ Calcium-channel blockers & 2.1893 & $0.975-5.026$ & $0.002 *$ \\
$\quad$ Statins & 3.8721 & $1.650-9.656$ & 0.29 \\
Laboratory data & & & $0.999-1.001$ \\
$\quad$ BNP & 1.0005 & & \\
\hline
\end{tabular}

$\mathrm{CI}$ indicates confidence interval; $\mathrm{ARB}$, angiotensin receptor blocker; and ACEI, angiotensin converting enzyme inhibitor.

ences between the patients with shorter and longer FCL in the univariate analysis, but the use of statins was the only significant determinant for longer FCL in the multivariate analysis. Finally, FCL was significantly longer in patients with statin prescription than in those without.
Detection of new-onset AF in clinical study: We focused on new-onset AF episodes in the present study. When considering the concept of the atrial remodeling in AF, it seems reasonable that AF patients with longer clinical histories should be influenced more strongly by the progression of atrial remodeling, 


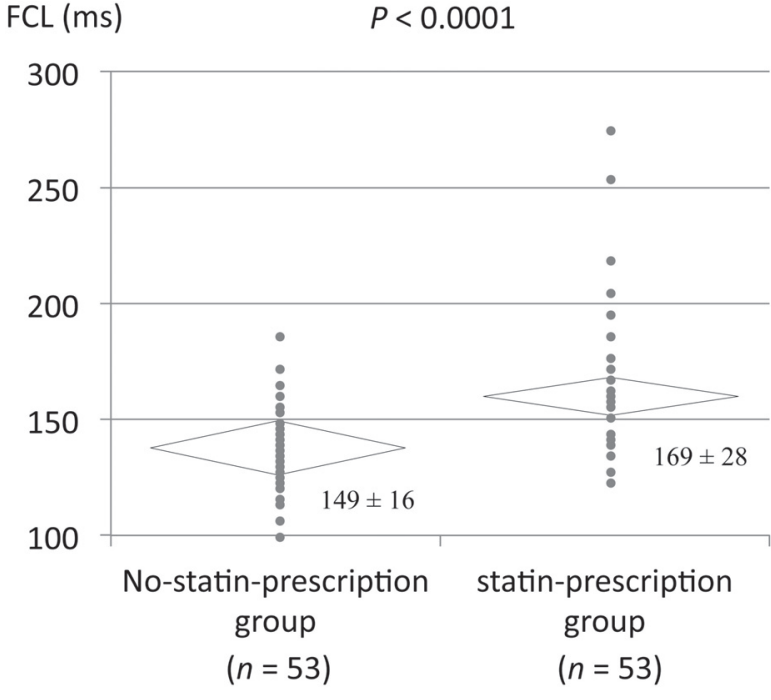

Figure 2. FCL data for the two groups. This figure displays FCL data for the two groups. In the graph, we have adjusted age with and without statin. Data are from 53 patients. The mean age was $71 \pm 8$ years. In the nostatin-prescription group, the mean FCL was $149 \pm 16 \mathrm{~ms}$. On the other hand, in the statin-prescription group, the mean FCL was $169 \pm 28 \mathrm{~ms}$. The FCL was significantly prolonged in the statin prescription group.

and the patients with new-onset AF, ie, those with the shortest clinical history of AF, may have atria that have been influenced less from the progression of atrial remodeling. ${ }^{17)}$ Because we hypothesized that various clinical factors, including basic disease and/or various prescribed medicines, may influence the progression of atrial remodeling, we compared various factors, including FCL, in patients with new-onset AF. Patients with new-onset AF were identified through the ECG storage system on the ECG server at our institute. Because most of the patients have several ECG recordings in this system, patients with permanent and long-lasting AF could be eliminated by referring to the preceding ECG recording. We also eliminated the patients with paroxysmal or persistent $\mathrm{AF}$ by referring to their clinical histories as well as preceding ECGs. This retrospective evaluation could be done because this study was conducted in a single institute. To the best of our knowledge, this was the first systematic detection of new-onset $\mathrm{AF}$ in clinical cases.

Evaluation of FCL in the surface ECG recording: Several studies have demonstrated the usefulness of FCL measurement in surface ECG recordings. We have also reported the usefulness of FCL measurement in the surface ECG as an indirect but reproducible evaluation of the atrial electrophysiological properties in $\mathrm{AF}^{12)}$ In the present study, we applied the same customized fibrillation wave analyzer to the ECG recordings stored in the ECG server and successfully determined FCL in patients with new-onset AF. As we have discussed in previous reports, FCL calculated through analysis of the surface ECG is an indirect parameter because it is influenced not only by the refractoriness but also the complexity or numbers of reentrant circuits of both atria in AF. ${ }^{18,19)}$ However, a rough but significant relationship between FCL and atrial refractoriness has been documented; therefore, the FCL shortening might be able to be used as a marker of the progression of atrial electrical remodeling. In the present study, longer FCL was correlated with the prescription of calcium channel blockers, ARBs/ACEIs or statins; therefore, we speculate that the atrial remodeling may have progressed less in patients with such prescriptions. In contrast, Yokokawa, et al have reported the relationship between higher age and more complex atrial substrate in patients undergoing ablation of persistent $\mathrm{AF}^{20)}$ Because such substrate was characterized by areas of low voltage/scar, and was associated with a lower AF frequency, it may lead to lower voltage of fibrillation waves in the surface ECG and may result in shorter FCL, which does not agree with the result of our present study. The reason for such a discrepancy is unclear, but modification of AF substrate caused by catheter ablation or by the passage of time might be different. Our data are compatible at least with the preceding papers which observed changes in FCL over time. $^{12,13)}$

Statins, possible prophylactic suppressors of the progression of atrial remodeling: In the present study which used an age-adjusted model, multivariate analysis indicated that the use of statins was the only significant determinant for longer FCL in patients with new-onset AF. Because aging is an important risk factor for the clinical appearance as well as progression of $\mathrm{AF}^{3)}$ the use of an age-adjusted model is necessary in this study. The significant correlation between statin use and longer FCL observed in this study may indicate a prophylactic effect of statins for FCL shortening, at least in the cases with newonset AF.

Clinical AF is considered to be caused by various factors, including hypertension, oxidative stress and/or inflammation. Chung, et al have reported that the level of C-reactive protein (CRP) was higher in AF patients than in patients with normal sinus rhythms, and that the CRP level was higher in patients with persistent $\mathrm{AF}$ than in those with paroxysmal $\mathrm{AF}^{21)}$ Several experimental studies have also demonstrated increased oxidative stress and the infiltration of macrophages into atrial tissue in an AF model. ${ }^{22,23)}$ These results indicate a causative role for inflammation of the atrial substrate in AF.

Statins, which inhibit 3-hydroxy-3-methyl-glutaryl coenzyme A reductase, suppress cholesterol synthesis through the mevalonate pathway, but they have also been known to exert various other pleiotropic effects, including anti-inflammatory, antioxidant, antithrombotic, and angiogenic effects, as well as improvement of vascular endothelial function. ${ }^{24-26)}$ In cases of $\mathrm{AF}$, statins may be expected to suppress atrial remodeling, but documentation of a clinical effect is still limited to cases of postoperative AF after cardiac surgery, ${ }^{27)}$ or the recurrence rate of AF after electrical cardioversion. ${ }^{28)}$ In the present study, we have documented a significant correlation between statin use and longer FCL, which may indicate a prophylactic suppression of the progression of atrial remodeling, at least in patients with new-onset AF. This may also indicate that such "upstream therapy" may be effective in cases of AF, in its extremely early phase of clinical appearance.

Limitations: This study has several limitations. First, our method of detecting new-onset AF may include errors related to the underestimation of preceding AF episodes. There were a considerable number of patients with "asymptomatic" AF in the study population, so the duration could not be identified in such patients. Second, because atrial refractoriness was only evaluated through the FCL measurement, the atrial refractoriness in patients with paroxysmal AF could not be evaluated when an ECG of AF was not available. Third, because this 
study had a retrospective design, the use of various medicines was not randomized. This was unavoidable because identification of new-onset AF patients could only be performed since this was a retrospective study at a single institute. Finally, the number of patients was limited and sampling bias could not be eliminated. These limitations should be solved in future studies with larger numbers of patients.

Conclusion: We detected patients with new-onset AF using our digital ECG storage system and evaluated FCL in the surface ECG recording. Multivariate analysis comparing patients with longer and shorter FCLs revealed a significant correlation between statin use and longer FCL. If we interpret the longer FCL as the marker of less progressed atrial remodeling, this result may indicate a prophylactic suppressive effect of statin on atrial remodeling in cases of new-onset AF.

\section{REFERENCES}

1. Nattel S, Opie LH. Controversies in atrial fibrillation. Lancet 2006; 367: 262-72. (Review)

2. Suzuki S, Yamashita T, Ohtsuka T, et al. Prevalence and prognosis of patients with atrial fibrillation in Japan: a prospective cohort of Shinken Database 2004. Circ J 2008; 72: 914-20.

3. Inoue $\mathrm{H}$, Fujiki A, Origasa $\mathrm{H}$, et al. Prevalence of atrial fibrillation in the general population of Japan: an analysis based on periodic health examination. Int J Cardiol 2009; 137: 102-7.

4. Kumagai K, Nakashime H, Urata H, Gondo N, Arakawa K, Saku $\mathrm{K}$. Effects of angiotensin II type 1 receptor antagonist on electrical and structural remodeling in atrial fibrillation. J Am Coll Cardio 2003; 41: 2197-204.

5. Nakashima H, Kumagai K, Urata H, Gondo N, Ideishi M, Arakawa K. Angiotensin II antagonist prevents electrical remodeling in atrial fibrillation. Circulation 2000; 101: 2612-7.

6. Shiroshita-Takeshita A, Brundel BJ, Burstein B, et al. Effects of simvastatin on the development of the atrial fibrillation substrate in dogs with congestive heart failure. Cardiovasc Res 2007; 74: $75-84$.

7. Kumagai K, Nakashima H, Saku K. The HMG-CoA reductase inhibitor atorvastatin prevents atrial fibrillation by inhibiting inflammation in a canine sterile pericarditis model. Cardiovasc Res 2004, 62: 105-11.

8. Patti G, Chello M, Candura D, et al. Randomized trial of atorvastatin for reduction of postoperative atrial fibrillation in patients undergoing cardiac surgery: results of the ARMYDA-3 (Atorvastatin for Reduction of MYocardial Dysrhythmia After cardiac surgery) study. Circulation 2006; 114: 1455-61.

9. Schneider MP, Hua TA, Böhm M, Wachtell K, Kjeldsen SE, Schmieder RE. Prevention of atrial fibrillation by renin-angiotensin system inhibition a meta-analysis. J Am Coll Cardiol 2010; 55: 2299-307. (Review)

10. Liu T, Li L, Korantzopoulos P, Liu E, Li G. Statin use and development of atrial fibrillation: a systematic review and meta-analysis of randomized clinical trials and observational studies. Int J Cardiol 2008; 126: 160-70. (Review)
11. Husser D, Stridh M, Sornmol L, Olsson SB, Bollmann A. Frequency analysis of atrial fibrillation from the surface electrocardiogram. Indian Pacing Electrophysiol J 2004; 4: 122-36.

12. Sasaki T, Niwano S, Sasaki S, et al. Long-term follow-up of changes in fibrillation waves in patients with persistent atrial fibrillation: spectral analysis of surface ECG. Circ J 2006; 70: 169-73.

13. Fujiki A, Nakagawa H, Sakabe M, et al. Spectral characteristics of human atrial fibrillation waves of the right atrial free wall with respect to the duration of atrial fibrillation and effect of class I antiarrhythmic drugs. Jpn Circ J 2001; 65: 1047-51.

14. Niwano S, Sasaki T, Kurokawa S, et al. Predicting the efficacy of antiarrhythmic agents for interrupting persistent atrial fibrillation according to spectral analysis of the fibrillation waves on the surface ECG. Circ J 2009; 73: 1210-8.

15. Fujiki A, Sakabe M, Nishida K, Mizumaki K, Inoue H. Role of fibrillation cycle length in spontaneous and drug-induced termination of human atrial fibrillation. Circ J 2003; 67: 391-5.

16. Newgard CD, Hedges JR, Arthur M, Mullins RJ. Advanced statistics: the propensity score -- a method for estimating treatment effect in observational research. Acad Emerg Med 2004; 11: 953-61.

17. Nattel S. New ideas about atrial fibrillation 50 years on. Nature 2002; 415: 219-26. (Review)

18. Neuberger HR, Schotten U, Blaauw Y, et al. Chronic atrial dilation, electrical remodeling, and atrial fibrillation in the goat. J Am Coll Cardiol 2006; 47: 644-53.

19. Watanabe I, Okumura Y, Nagashima K, et al. Electrical remodeling in fibrillating canine atrium: action potential alternans during rapid atrial pacing and late phase 3 early afterdepolarization after cessation of rapid atrial pacing. Int Heart J 2010; 51: 354-8.

20. Yokokawa M, Latchamsetty R, Good E, et al. The impact of age on the atrial substrate: insights from patients with a low scar burden undergoing catheter ablation of persistent atrial fibrillation. J Interv Card Electrophysiol 2012; 34: 287-94.

21. Chung MK, Martin DO, Sprecher D, et al. C-reactive protein elevation in patients with atrial arrhythmias: inflammatory mechanisms and persistence of atrial fibrillation. Circulation 2001; 104: 2886-91.

22. Geo G, Dudley SC Jr. Redox regulation, NF-kappaB, and atrial fibrillation. Antioxid Redox Signal 2009; 11: 2265-77. (Review)

23. Shiroshita-Takeshita A, Schram G, Lavoie J, Nattel S. Effect of simvastatin and antioxidant vitamins on atrial fibrillation promotion by atrial-tachycardia remodeling in dogs. Circulation 2004; 110: 2313-9.

24. Sacks FM. High-intensity statin treatment for coronary heart disease. JAMA 2004; 291: 1132-4.

25. Ridker PM, Rifai N, Clearfield M, et al. Measurement of C-reactive protein for the targeting of statin therapy in the primary prevention of acute coronary events. N Engl J Med 2001; 344: 195965.

26. Shishehbor MH, Brennan ML, Aviles RJ, et al. Statins promote potent systemic antioxidant effects through specific inflammatory pathways. Circulation 2003; 108: 426-31.

27. Ji Q, Mei Y, Wang X, et al. Effect of preoperative atorvastatin therapy on atrial fibrillation following off-pump coronary artery bypass grafting. Circ J 2009; 73: 2244-9.

28. Ozaydin M, Varol E, Aslan SM, et al. Effect of atorvastatin on the recurrence rates of atrial fibrillation after electrical cardioversion. Am J Cardiol 2006; 97: 1490-3. 